\title{
The internal mammary artery perforator flap and its subtypes in the reconstruction of median sternotomy wounds
}

\author{
Ruben Y. Kannan, MB, PhD, FRCS(Plast)
}

\section{ABSTRACT}

Objective: To determine the feasibility of using the internal mammary artery perforator (IMAP) flap for superficial and deep sternal wound breakdowns.

Methods: This was a retrospective case review of 9 patients with sternal wound dehiscence over an 18-month period between 2013 and 2015. Seven of the 9 patients received a single IMAP flap to cover full-length sternal wounds, including 4 with a fasciocutaneous flap and 3 with a musculocutaneous flap.

Results: All of the patients were male, with a mean age of 68 years. The mean number of perforators was 1.3 , with a mean perforator diameter of $1.5 \mathrm{~mm}$. In all cases, the torsion angle was 80 degrees, with a translational pedicle movement of 1 to $2 \mathrm{~cm}$. There were no instances of total flap failure and only 2 cases of partial flap necrosis, which were managed conservatively. One flap, performed when both internal mammary arteries had been harvested previously, showed complete survival.

Conclusions: The IMAP flap has an advantage in its the ability to reconstruct the entire length of a sternotomy wound from the suprasternal notch to the xiphisternum with relatively minimal dissection and morbidity compared with more conventional flaps such as pectoralis major, rectus, and omental flaps. Nevertheless, caveats for its use remain, such as in patients with vasopressor therapy and the resulting subclavicular scar, which is unaesthetic in women. Overall, the IMAP flap is an attractive reconstructive tool specifically in stable male patients with noninfected sternotomy wound dehiscence with a defect width of up to $7 \mathrm{~cm}$. In this patient subset, it is the ideal first-line reconstructive tool. (J Thorac Cardiovasc Surg 2016;152:264-8)

Sternotomy wound breakdown following cardiac surgery is an uncommon but serious event that can leave significant defects, often exposing the mediastinum. ${ }^{1}$ A common cause is wound infection, but occasionally mechanical dehiscence of the skin can occur, particularly in conditions involving increased chest wall excursion (eg, patient struggling to breathe). The standard first line of management is surgical debridement, followed by the removal of sternal wires, if necessary. Once the wound is clean, topical negative pressure therapy is applied until definitive closure is achieved.

From the Department of Plastic Surgery, Queen Elizabeth Hospital, Birmingham, United Kingdom.

Received for publication Nov 26, 2015; revisions received Jan 21, 2016; accepted for publication Jan 26, 2016; available ahead of print March 18, 2016.

Address for reprints: Ruben Y. Kannan, MB, PhD, FRCS(Plast), Department of Plastic Surgery, Queen Elizabeth Hospital, 5 Renishaw Drive, Leicester LE5 5TY, UK (E-mail: ruben.kannan @ gmail.com).

$0022-5223 / \$ 36.00$

Copyright $\odot 2016$ by The American Association for Thoracic Surgery

http://dx.doi.org/10.1016/j.jtcvs.2016.01.053

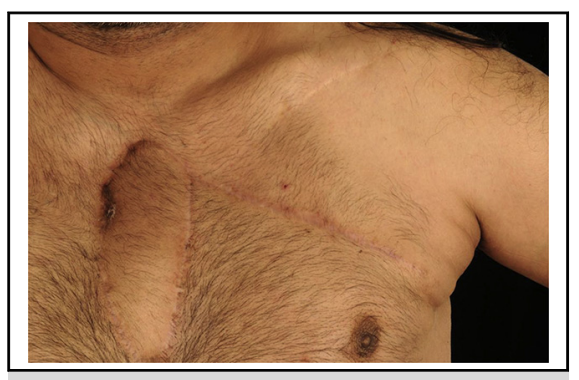

IMAP flap reconstruction for sternal wounds.

\section{Central Message}

The IMAP flap is a niche flap with specific indications in sternal wound reconstruction and, when appropriate, provides a relatively simple solution to a complex problem.

\section{Perspective}

IMAP flap reconstruction will mark a drastic change in how sternal wounds are approached.

See Editorial Commentary page 269.

At this stage, tissue must be imported into the wound in the form of a flap to achieve wound healing. Conventional reconstructive flaps used in sternal reconstruction, including pectoralis major, rectus, and omental flaps, either do not cover the entire length of the defect or, as in the case of the omental flap, necessitate laparotomy.

Although useful for closing voluminous defects, each single flap does not have the capacity to resurface the entire length of the sternal defect, and flaps must be used in tandem to close these wounds, as evidenced by the anatomic classifications of sternal defects aligned closely with muscle flap selection. ${ }^{3}$ Moreover, sacrificing these muscles

Scanning this QR code will take you to the article title page.

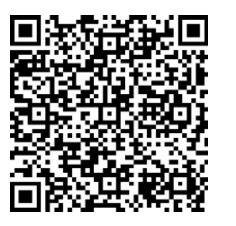




\section{Abbreviations and Acronyms \\ ASA $=$ American Society of Anesthesiologists \\ $\mathrm{CABG}=$ coronary artery bypass grafting \\ CT $=$ computed tomography \\ DSEP = deep superior epigastric perforator \\ fc-IMAP $=$ fasciocutaneous IMAP \\ IMA $=$ internal mammary artery \\ IMAP $=$ internal mammary artery perforator \\ LTAP $=$ lateral thoracic artery perforator \\ mc-IMAP $=$ musculocutaneous IMAP}

can cause functional disability, potential hernias (in the case of the rectus muscle), seromas, and other problems. In addition, such extensive chest muscle dissection at that depth can restrict chest excursions in the postoperative period due to pain.

Other treatment options include the use of topical negative pressure therapy ${ }^{4}$ to eventually close these wounds; however, closure takes a long time, with the potential for infection. More recently, the deep superior epigastric perforator (DSEP) flap has been described for sternal wound closure. ${ }^{5,6}$ This fasciocutaneous perforator flap, based on the single perforator of the DSEP, pivoted through 90 degrees, has the capability of successfully resurfacing large defects. Reported advantages include relatively less dissection, muscle function preservation, and potentially a hidden submammary scar in women. However, use of the DSEP flap requires an intact internal mammary artery (IMA) axis, is limited by the potential length that can be harvested, and, if a deeper mediastinal defect is to be reconstructed, the lack of additional muscle beneath the DSEP flap that can be included as a composite muscle-fasciocutaneous flap for filling dead space.

Here we describe the use of the internal mammary artery perforator (IMAP) flap in a spectrum of presternal and mediastinal defects as an alternative form of reconstruction in selected cases. Its major advantage is that a single fasciocutaneous or musculocutaneous IMAP flap can cover any 3-dimensional sternotomy wound defect.

\section{PATIENTS AND METHODS}

In a retrospective case series over an 18-month period between 2013 and 2015, a total of 9 patients requiring midline chest reconstruction were referred, of which 7 had an IMAP flap. The remaining 2 patients included 1 patient with a pectoralis major muscle advancement flap and 1 patient who received topical negative pressure therapy. All 9 patients were referred from the cardiothoracic surgical unit at the Queen Elizabeth Hospital following wound breakdown after cardiothoracic surgery.

All patients were assessed initially for suitability for the IMAP flap using a hand-held 8- MHz Doppler probe (Huntleigh, Cardiff, United Kingdom). Preoperative perforator selection in terms of size and site was confirmed using computed tomography (CT) angiography, where appropriate. In 1 patient, CT angiography was not performed because both internal mammary arteries were previously harvested for coronary bypass procedures.

The 9 patients in this cohort included 7 males and 2 females, with a mean age of 50 years (range, 30 to 75 years). The most common comorbidities were hypertension, diabetes, ischemic heart disease, and immunosuppression (in the transplant recipients). One patient suffered from cystic fibrosis and had undergone heart-lung transplantation. Overall premorbid status was reflected in the American Society of Anesthesiologists (ASA) grade; 3 patients were ASA grade 2, 4 patients were ASA grade 3, and 2 patients were ASA grade 4. In this cohort, premorbid status was then weighted as part of the decision making process, with the following exclusion criteria: (1) receipt of vasopressors or at high probability of receiving vasopressors postoperatively, particularly in patients who were already septic; (2) ongoing local infection (eg, osteomyelitis even after multiple debridements); and (3) ASA grade of $\geq 4$.

Two of the 9 patients were excluded, 1 who was already on vasopressors and thus received a pectoralis major muscle advancement flap, and 1 who had poor wound healing due to a persistent infection, which ruled out the IMAP flap as a reconstructive option. This patient was left to heal secondarily with the aid of topical negative pressure therapy. Both of these patients had an ASA grade of 4.

\section{Surgical Technique}

Based on the CT angiogram, the most optimal IMA perforator, usually the second but occasionally the first or third, is identified with the Doppler probe preoperatively and marked. Next, the lateral thoracic artery perforator (LTAP) is marked, and the IMAP-LTAP axis is drawn out. The fasciocutaneous IMAP (fc-IMAP) flap is raised subfascially up to the mid-axillary line. If required, axillary lymph nodes can be included within the flap as a chimeric-in-parallel component, to reduce the risk of lymphoedema and a subsequent "pin-cushioning" effect within the propeller flap. The maximum length of the flap can be up to $25 \mathrm{~cm}$, and provided that the width of IMAP flap is kept below $7 \mathrm{~cm}$, the secondary defect can be closed primarily. If possible, superficial veins at the pivot point are preserved to maximize venous outflow.

For deeper mediastinal defects, a musculocutaneous IMAP (mc-IMAP) flap can be raised with the underlying pectoralis major muscle for additional bulk and volume filling. Use of an mc-IMAP flap also provides a longer vertical length of the IMA perforator to be included within the composite flap, as well as an increased perforator diameter as the pivot point is closed to the internal mammary vessels. For additional perfusion, a 1-cm cuff of pectoralis muscle is preserved around the fc-IMAP; this modification has been found to augment flap perfusion and increase its dimensions.

Postoperatively, the patient is kept ventilated overnight in the intensive care unit, with a reduced chest wall ventilatory pattern maintained by a cardiac anesthesist. Flap observations are continued hourly for the first 24 hours and every 2 hours on the second postoperative day. Chest physiotherapy is instituted after 48 hours. After 5 days, the patient is given chest wall splints and discharged to home.

\section{RESULTS}

Of the 7 patients who received an IMAP flap, 4 had an fc-IMAP flap and 3 had an mc-IMAP flap. All 7 patients were male, with a mean age of 68 years (range, 44 to 79 years). The average defect size was $21 \times 5 \mathrm{~cm}$. All of these defects encompassed the full length of the sternum, with the fc-IMAP flap used for presternal defects and the mc-IMAP flap used for deeper mediastinal wounds. 
The mean number of perforators within the IMAP flap was 1.3 , with a mean perforating vessel diameter of $1.5 \mathrm{~mm}$. Because the IMAP flap was designed along the IMAP-LTAP axis, the angle of torsion was 80 degrees in these patients.

There were no instances of postoperative mortality, and all of the flaps survived, with 2 cases of partial flap failure occurring in the fc-IMAP group. In these cases, although the distal third of the flap skin necrosed, there was viable adipose tissue beneath, which healed secondarily. One of these cases was due to the pressure effects of an underlying postoperative abscess, whereas the other was secondary to postoperative vasopressor use. Both of these cases were in the early part of the study period and did not involve the use of a "muscle cuff" around the IMAP flap. The details of some individual cases are presented below.

\section{Case 1}

This 60-year-old man, a known smoker, presented with sternal osteomyelitis with culture-proven fungal infection and wound breakdown after cardiac surgery. He underwent multiple wound debridement, including the removal of all sternal wires until tissue cultures were negative. Preoperative chest CT showed no further evidence of osteomyelitis. His $19 \times 4 \mathrm{~cm}$ wound was subsequently reconstructed with a right-sided second IMAP fasciocutaneous flap. The distal $20 \%$ of the flap was found to have sustained superficial necrosis at 1 week postoperatively secondary to a tight collection beneath the flap. Release of the deep sutures revealed a collection of pus beneath the flap, which hindered wound healing in the distal $4 \mathrm{~cm}$ of the sternotomy wound over the subsequent few weeks. The distal part of the flap was then debrided, and a split-skin graft was applied. This eventually healed, but the patient was left with a few sinuses from a persistent fungal infection. This was managed conservatively with long-term antibiotic therapy.

\section{Case 2}

This 45-year-old man who had undergone coronary artery bypass grafting (CABG) 4 months earlier developed a presternal wound breakdown measuring $20 \times 6 \mathrm{~cm}$ from the suprasternal notch to the xiphisternum. In this case, the entire length of the IMAs had been harvested, and thus the use of a pectoralis major or rectus muscle flap was not considered. Doppler studies revealed evidence of perforator signals on both sides of the sternum at the second rib level despite the absence of the IMAs. Based on this finding, intraoperative exploration of the wound was performed, which confirmed the presence of flow from deep to superficial planes within the IMAP flap area. The flap was designed with its axis from the IMAP to the LTAP and raised in

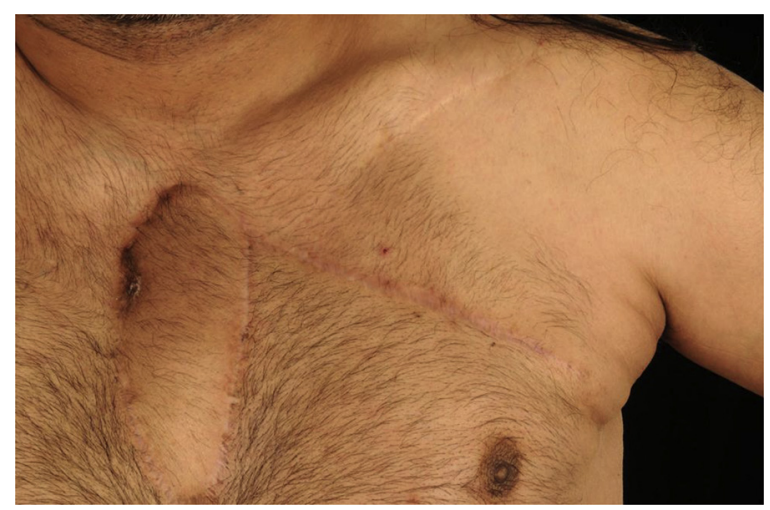

FIGURE 1. Eight-month postoperative image of a healed IMAP flap in a patient with previously harvested bilateral IMAs.

the subfascial plane to include the deeper vascular plexuses within it. Because the IMAs had been harvested 4 months earlier, this effectively amounted to a "delay-type" procedure and accounted for the flow of perfusion from the LTAP to the IMAP via the deeper vascular networks beneath the fascia. The wound healed within 10 days, and the patient remained well at 8 months after surgery (Figure 1).

\section{Case 3}

This 79-year-old man developed sternotomy wound breakdown following a CABG procedure and was unwell. He had a presternal wound extending the full length of the sternum, with a defect size of $17 \times 4 \mathrm{~cm}$. He received an IMAP flap based on the second intercostal perforator, but developed a chest infection within 72 hours of reconstruction, followed by sepsis. This necessitated vasopressor use on the intensive therapy unit/intensive care unit, which resulted in superficial necrosis of the distal third of the IMAP flap over the next 2 weeks, which was managed conservatively.

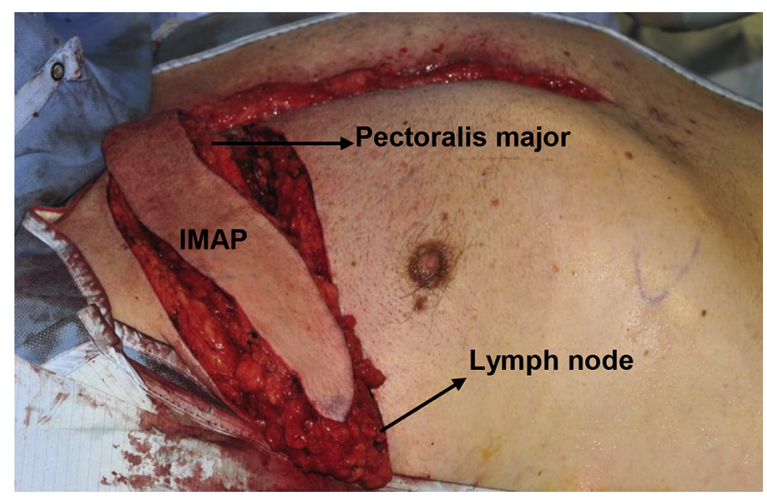

FIGURE 2. Perioperative view of a musculocutaneous IMAP flap used for closure of a deep mediastinal wound with a chimeric-in-parallel lymph node component within it. IMAP, Internal mammary artery perforator. 


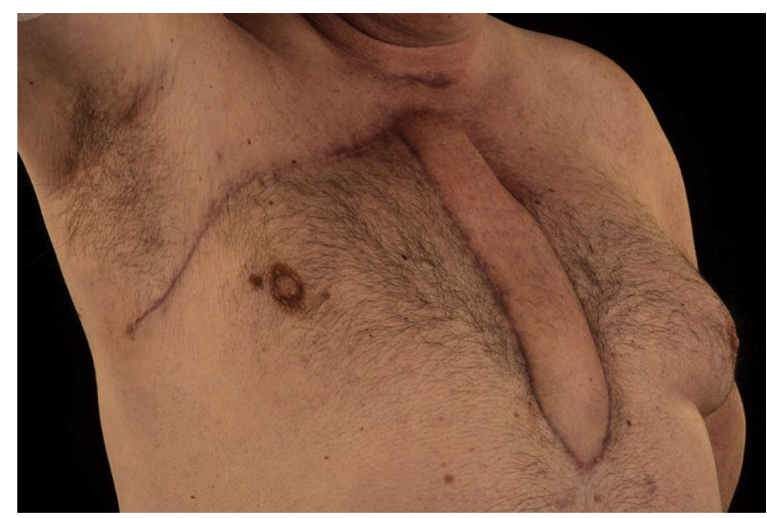

FIGURE 3. Two-month postoperative image of a healed mc-IMAP flap with a chimeric lymph node component in situ. Donor site morbidity was minimal.

\section{Case 4}

This 49-year-old obese man who had undergone a CABG procedure experienced breakdown at 2 weeks postsurgery, with a deep, but clean, $20 \times 4 \mathrm{~cm}$ defect down to the anterior mediastinum. A preoperative CT angiogram identified the right first and second IMAPs as the most optimal perforators, and thus an IMAP flap was raised based on these perforators. Given the depth of the defect, this IMAP flap was raised with its underlying pectoralis major muscle as a composite flap, with an axillary lymph node within it to reduce lymphoedema within the flap (Figure 2). The patient was discharged from the hospital within 10 days of the procedure. Imaging at 2 months postsurgery showed a healed wound (Figure 3). The patient experienced no functional morbidity associated with losing a strip of the pectoralis major muscle.

The overall results for our patient cohort summarized in Table 1. A preliminary glance at the data suggests that IMAP flaps tend to perform better in relatively healthier patients with an ASA grades of $\leq 3$. Even so, one-half of our patients with ASA grade 3 developed partial flap failure, whereas all of the patients with ASA grade 2 had an optimal outcome. This is perhaps an indirect reflection of the need for optimal intrinsic healing and vascularity to ensure the best reconstructive outcomes. The development of recurrent infections despite flap reconstruction also underscores the importance of adequate surgical debridement for the overall outcome.

\section{DISCUSSION}

The IMAP flap was first described as a perforator flap based on the deltopectoral axis to close tracheostomal defects. ${ }^{7}$ Subsequent studies have shown its benefit for the coverage of small- to medium-sized chest wall wounds after mastectomy. ${ }^{8}$ In this study, we further pushed the boundaries of the IMAP flap for sternal wound dehiscence by using it as a fasciocutaneous flap for wounds in which the sternum itself was intact and as a musculocutaneous flap for mediastinal defects. Initial results showed that the fc-IMAP flap, although providing a better contoured fit for presternal wounds, was more susceptible to pedicle torsion and thus distal venous compromise, despite preoperative perforator localization with CT angiography. Interestingly, the fcIMAP flap that was delayed survived completely with no postoperative complications, possibly because the IMA perforator diameter increased in size correspondingly over time.

The success rates of the mc-IMAP flap versus the fcIMAP flap cannot be compared in this study, given the small number of patients. Nonetheless, circumstantial evidence from the Kuopio group suggests that the mc-IMAP variant does better. ${ }^{9}$ A possible explanation for this is that

TABLE 1. Demographic profile and outcomes of the midline sternal wound reconstruction in our patient cohort

\begin{tabular}{|c|c|c|c|c|c|c|c|c|}
\hline Sex & Age, $\mathbf{y}$ & Original procedure & Comorbidities & Defect & Reconstruction & $\begin{array}{c}\text { ASA } \\
\text { grade }\end{array}$ & $\begin{array}{c}\text { Reconstructive } \\
\text { outcome }\end{array}$ & Overall outcome \\
\hline Male & 44 & CABG & DM, HT, IHD & Presternal & IMAP & 2 & Success & Healed wound \\
\hline Female & 67 & CABG & $\begin{array}{l}\text { DM, HT, IHD, } \\
\text { immunosuppression, obesity }\end{array}$ & Mediastinal & $\mathrm{PM}$ & 4 & Success & Died from sepsis \\
\hline Female & 30 & Cardiac transplantation & $\begin{array}{l}\text { DM, ACS, IHD, fungal infection, } \\
\text { immunosuppression }\end{array}$ & Mediastinal & TNP & 4 & $\begin{array}{c}\text { Nonhealing } \\
\text { wound }\end{array}$ & Died from sepsis \\
\hline Male & 70 & CABG & DM, HT, IHD, fungal infection & Presternal & IMAP & 3 & Partial success & Recurrent infection \\
\hline Male & 75 & Cardiac transplantation & HT, DCM, immunosuppression & Presternal & IMAP & 3 & Partial success & Healed wound \\
\hline Male & 51 & Cardiac transplantation & $\begin{array}{l}\text { Cystic fibrosis, } \\
\text { immunosuppression }\end{array}$ & Presternal & IMAP & 2 & Success & Healed wound \\
\hline Male & 49 & Cardiac transplantation & HT, ACS, immunosuppression & Mediastinal & IMAP & 3 & Success & Healed wound \\
\hline Male & 54 & Cardiac transplantation & HT, IHD, immunosuppression & Mediastinal & IMAP & 3 & Success & Recurrent infection \\
\hline Male & 55 & Cardiac transplantation & HT, IHD, immunosuppression & Mediastinal & IMAP & 2 & Success & Healed wound \\
\hline
\end{tabular}

$A S A$, American Society of Anesthesiologists; $C A B G$, coronary artery bypass graft; $D M$, diabetes mellitus; $H T$, hypertension; $I H D$, ischemic heart disease; $I M A P$, internal mammary artery perforator flap; $P M$, pectoralis major muscle flap; $A C S$, acute coronary syndrome; $T N P$, topical negative-pressure therapy; $D C M$, dilated cardiomyopathy. 
because the IMA perforator suprafascial diameter is smaller than its deeper submuscular segment, when torsion is to be applied, the effects would be least on the perforator at its largest diameter, that is, at a deeper plane. Schelleken and colleagues ${ }^{10}$ in an anatomic study, showed that second IMAP length could be almost doubled when traced toward its origin, and correspondingly so for the first and third IMAPs. Thus, further perforator dissection would increase perforator length and further reduce pedicle torsion. Alternatively, leaving a cuff of pectoralis major muscle when dissecting the perforator down to the IMA would have the same effect, but with a reduced risk of perforator damage. This technical modification would improve survival rates for the fc-IMAP flap.

\section{CONCLUSIONS}

The IMAP flap is ideal for sternal wound reconstruction in male patients in stable condition with a noninfected wound and a defect width of up to $7 \mathrm{~cm}$. Larger defects will require skin grafting, which will leave an unaesthetic scar across the chest. Although the associated scar is not ideal in younger women, it may be tolerated in the more elderly women with ptotic breasts. The other prerogative is a surgeon with a high level of microsurgical expertise. In this scenario, the IMAP flap can be considered the first-line option, with the conventional muscle flaps kept in reserve in the event of suboptimal results.

\section{Conflict of Interest Statement}

Author has nothing to disclose with regard to commercial support.

\section{References}

1. Cohen M, Silverman NA, Goldfaden DM, Levitsky S. Reconstruction of infected median sternotomy wounds. Arch Surg. 1987;122:323-7.

2. Jurkiewicz MJ, Bostwick J III, Hester TR, Bishop JB, Craver J. Infected median sternotomy wound. Successful treatment by muscle flaps. Ann Surg. 1980;191: 738-44.

3. Greig AV, Geh JL, Khaduja V, Shibu M. Choice of flap for the management of deep sternal wound infection: an anatomical classification. J Plast Reconstr Aesthet Surg. 2007;60:372-8.

4. Falagas ME, Tansarli GS, Kapaskelis A, Vardakas KZ. Impact of vacuumassisted closure (VAC) therapy on clinical outcomes of patients with sternal wound infections: a meta-analysis of non-randomized studies. PLoS One. 2013;8:e64741

5. Oni G, Sharma R, Rao R, Unger J, Saint-Cyr M. Bilateral superior epigastric pedicle perforator flaps for total chest wall coverage. J Plast Reconstr Aesthet Surg. 2011;64:1104-7.

6. Wettstein R, Weisser M, Schaefer DJ, Kalbermatten DF. Superior epigastric artery perforator flap for sternal osteomyelitis defect reconstruction. J Plast Reconstr Aesthet Surg. 2014;67:634-9.

7. Yu P, Roblin P, Chevray P. Internal mammary artery perforator (IMAP) flap for tracheostoma reconstruction. Head Neck. 2006;28:723-9.

8. Takeuchi M, Sakurai H. Internal mammary artery perforator flap for reconstruction of the chest wall. J Plast Surg Hand Surg. 2013;47: 328-30.

9. Berg LT, Jaakkola P. Kuopio treatment strategy after deep sternal wound infection. Scand J Surg. 2014;102:3-8.

10. Schellekens PP, Paes EC, Hage JJ, van der Wal MB, Bleys RL, Kon M. Anatomy of the vascular pedicle of the internal mammary artery perforator (IMAP) flap as applied for head and neck reconstruction. J Plast Reconstr Aesthet Surg. 2011;64:53-7.

Key Words: internal mammary artery perforator flap, median sternotomy wound 